\title{
ON THE FREQUENCY OF PAIRS OF SQUARE-FREE NUMBERS WITH A GIVEN DIFFERENCE
}

\author{
L. MIRSKY
}

If $k$ is a positive integer, then the function

$$
f(x)=f(x, k)=\sum_{n \leqq x}|\mu(n) \mu(n+k)|
$$

enumerates the number of pairs of square-free integers with fixed difference $k$ such that the smaller of the two does not exceed $x$. The purpose of the present note is to establish the following result.

Theorem. As $x \rightarrow \infty$ we have

$$
f(x)=\prod_{p}\left(1-\frac{2}{p^{2}}\right) \prod_{p^{2} \mid k}\left(1+\frac{1}{p^{2}-2}\right) x+O\left(x^{2 / 3} \log ^{4 / 3} x\right),
$$

where the O-constant may depend upon $k$.

In a previous publication ${ }^{1}$ I considered the more general sum

$$
F(x)=\sum_{n \leqq x} \mu_{r}\left(n+k_{1}\right) \cdots \mu_{r}\left(n+k_{s}\right),
$$

where $k_{1}, \cdots, k_{s}$ are distinct integers, $r$ is an integer greater than 1 , and $\mu_{r}(n)$ is defined as 0 or 1 according as $n$ is or is not divisible by the $r$ th power of a prime. I showed that, for $x \rightarrow \infty$,

$$
F(x)=A x+O\left(x^{2 /(r+1)+\epsilon)},\right.
$$

where $A$ is a constant which can be expressed as an infinite series or else as a product ranging over primes. The asymptotic formula (1) generalized and sharpened an earlier estimate due to Pillai. ${ }^{2}$ The present note furnishes a slight improvement on (1) for the case $r=2$, $s=2$. The factor $x^{6}$ in (1) arose from the expression $\max _{\nu \leqq x} d(\nu)$, and could not, therefore, be replaced by a power of $\log x$ by the method previously used.

Our notation is as follows. The letters $x, y$ denote positive numbers; all other small letters denote positive integers unless otherwise stated, and $p$ is reserved for primes.

The $O$-notation refers to the passage $x \rightarrow \infty$, and $O$-constants de-

Received by the editors July 8, 1948.

${ }^{1}$ L. Mirsky, Note on an asymptotic formula connected with r-free integers, Quart. J. Math. Oxford Ser. vol. 18 (1947) pp. 178-182.

${ }^{2}$ S. S. Pillai, On sets of square-free integers, J. Indian Math. Soc. N.S. vol. 2 (1936) pp. 116-118. 
pend at most upon $k$.

$(a, b)$ denotes the highest common factor of $a$ and $b$.

$d(n)$ denotes the number of divisors of $n$.

We write

$$
N_{1}(x, a, b, k)=\sum_{a u-b v=k, b v \leqq x} 1 ; \quad N_{2}(x, a, b, k)=\sum_{a u^{2}-b v^{2}=k, b v^{2} \leqq x} 1 .
$$

We begin with two preliminary estimates.

LEMmA 1. If $(a, b) \nmid k$, then $N_{1}(x, a, b, k)=0$.

If $(a, b) \mid k$, then $N_{1}(x, a, b, k)=x(a, b) / a b+O(1)$.

This result is effectively case $s=2$ of Lemma 2 of my paper referred to above. The proof is extremely simple and may be left to the reader.

Lemma 2. $N_{2}(x, a, b, k)=O(\log x)$.

For the case when $a b$ is not a square it was shown by Estermann ${ }^{3}$ that

$$
N_{2}(x, a, b, k) \leqq 2 d(k)\{\log (x+k)+1\} .
$$

If, on the other hand, $a b$ is a square, the required result follows trivially. For write $a=l^{2} t, b=m^{2} t$. If $t \nmid k$, then $N_{2}(x, a, b, k)=0$, while if $t \mid k$, then

$$
\begin{aligned}
N_{2}(x, a, b, k) & =N_{2}\left(x t^{-1}, l^{2}, m^{2}, k t^{-1}\right) \\
& \leqq \sum_{l^{2} u^{2}-m^{2} v^{2}=k t-1} 1 \leqq \sum_{r^{2}-s^{2}=k t-1} 1=O(1) .
\end{aligned}
$$

We now come to the proof of the theorem. Since

$$
|\mu(n)|=\sum_{m^{2} \mid n} \mu(m)
$$

we have

$$
f(x)=\sum_{a^{2} c-b^{2}} \mu(a) \mu(b)=\sum_{1}+\sum_{2},
$$

say, where $a b \leqq y$ in $\sum_{1}$ and $a b>y$ in $\sum_{2}$. Here $y$ denotes a function of $x$ to be fixed later.

Writing

$$
K=\sum_{(a, b)^{2} \mid k} \mu(a) \mu(b) \frac{(a, b)^{2}}{a^{2} b^{2}}
$$

${ }^{3}$ T. Estermann, Einige Sätze ilber quadratfreie Zahlen, Math. Ann. vol. 105 (1931) pp. 653-662, Hilfssatz 2 (Anhang). 
we have, by Lemma 1 ,

$$
\begin{aligned}
\sum_{1} & =\sum_{a b \leqq y} \mu(a) \mu(b) N_{1}\left(x, a^{2}, b^{2}, k\right) \\
& =x \sum_{a b \leqq y,\left(a^{2}, b^{2}\right) \mid k} \mu(a) \mu(b) \frac{(a, b)^{2}}{a^{2} b^{2}}+O(y \log y) \\
& =K x+O\left(x \sum_{a b>y} \frac{1}{a^{2} b^{2}}\right)+O(y \log y) \\
& =K x+O\left(x y^{-1} \log y\right)+O(y \log y) .
\end{aligned}
$$

Again, by Lemma 2,

$$
\begin{aligned}
\left|\sum_{2}\right| & \leqq \\
\sum_{a^{2} c-b^{2} d=k, b^{2} d \leqq x, a b>y} 1 \leqq & \sum_{a^{2} c-b^{2} d=k, b^{2} d \leqq x, c d<x(x+k) y^{-2}} 1 \\
& =\sum_{c d<x(x+k) y^{-2}} N_{2}(x, c, d, k)=O\left(x^{2} y^{-2} \log ^{2} x\right) .
\end{aligned}
$$

Putting $y=x^{2 / 3} \log ^{1 / 3} x$ we obtain, by (2), (3), and (4),

$$
f(x)=K x+O\left(x^{2 / 3} \log ^{4 / 3} x\right) .
$$

Finally, it is clear that $K=\prod_{p} \chi_{p}$, where

$$
\chi_{p}=\sum_{u, v=0,1 ;\left(p^{u}, p^{v}\right)^{2} \mid k} \mu\left(p^{u}\right) \mu\left(p^{v}\right) \frac{\left(p^{u}, p^{v}\right)^{2}}{p^{2 u+2 v}}=\left\{\begin{array}{lll}
1-1 / p^{2} & \text { if } & p^{2} \mid k, \\
1-2 / p^{2} & \text { if } & p^{2} \nmid k .
\end{array}\right.
$$

The theorem now follows at once.

It may be worth mentioning that the method of the present note also enables us to investigate the sums

$$
\sum_{n \leqq x}\left|\mu\left(q_{1} n+k_{1}\right) \mu\left(q_{2} n+k_{2}\right)\right|
$$

and

$$
\sum_{n \leqq x}\left|\mu\left(\left(q_{1} n+k_{1}\right)\left(q_{2} n+k_{2}\right)\right)\right|
$$

(where $k_{1}, k_{2}, q_{1}, q_{2}$ are given integers), and to obtain asymptotic formulae for these sums with error terms of the form $O\left(x^{2 / 3} \log ^{4 / 3} x\right)$.

Another result which can be established by an argument analogous to that used above is as follows.

Let $t_{1}(n)=O\left(\log ^{\alpha_{1}} n\right), t_{2}(n)=O\left(\log ^{\alpha_{2}} n\right)$, where $\alpha_{1}, \alpha_{2}$ are any given real numbers, and let

$$
T_{1}(n)=\sum_{m^{2} \mid n} t_{1}(m), \quad T_{2}(n)=\sum_{m^{2} \mid n} t_{2}(m) .
$$


Then

$$
\sum_{n \leqq x} T_{1}(n) T_{2}(n+k)=C x+O\left(x^{2 / 3} \log ^{\alpha_{1}+\alpha_{2}+4 / 3} x\right)
$$

where

$$
C=\sum_{(a, b)^{2} \mid k} t_{1}(a) t_{2}(b) \frac{(a, b)^{2}}{a^{2} b^{2}} .
$$

The theorem we have proved in detail is the special case $t_{1}(n)$ $=t_{2}(n)=\mu(n)$ of this result.

Added in proof (September 1949). In a recent paper (Quart. J. Math. Oxford Ser. vol. 20 (1949) pp. 65-79) F. V. Atkinson and Lord Cherwell obtained a generalization of Lemma 2 in which squares are replaced by $r$ th powers. This new estimate enables me to extend the result of the present paper to numbers not divisible by $r$ th powers, and to prove that, for any $r \geqq 2$,

$$
\begin{aligned}
\sum_{n \leqq x} \mu_{r}(n) \mu_{r}(n+k)=\prod_{p} & \left(1-\frac{2}{p^{r}}\right) \prod_{p^{r} \mid k}\left(\frac{p^{r}-1}{p^{r}-2}\right) x \\
& +O\left(x^{2 /(r+1)}(\log x)^{(r+2) /(r+1)}\right) .
\end{aligned}
$$

UNIVERSITY OF SHEFFIELD 